\title{
Análisis Costo-Beneficio para la Zona Costera Norte en la Provincia de Camagüey, Cuba
}

\author{
Análise Custo-Benefício para o Litoral Norte da Provincia de Camagüey, Cuba \\ Cost-Benefit Analysis for Northern Coastal Zone of Camagüey Province, Cuba
}

\author{
María Elena Zequeira Álvarez ${ }^{1}$ \\ Ernesto Figueredo Castellanos ${ }^{2}$ \\ Arístides Pelegrín Mesa ${ }^{3}$ \\ Alain Hernández Santoyo ${ }^{4}$ \\ Silvina Beatriz Varona Reyes ${ }^{5}$
}

Artigo recebido para publicação em Jul/2013 e aceito para publicação em Ago/2013.

\section{RESUMO}

No litoral norte da província de Camagüey encontrasse um importante plano de desenvolvimento turístico a médio prazo. A área de estudo é composta por $4.062,0 \mathrm{~km}^{2}$ (terra firme, ilhotas e área marinha) e limita-se a curva de nível de 5 metros. É um ecossistema muito frágil, pois há dez áreas protegidas, um deles com importância internacional (Reserva de Fauna "Río Máximo" de Camagüey). O objetivo deste trabalho é analisar a relação custo-benefício para a área costeira do norte da província de Camagüey, Cuba. Utilizasse o procedimento metodológico projetado e validado no Projeto Territorial "Análise da produção de bens e serviços ambientais na área costeira norte da província de Camagüey, Cuba" executado pelo CIMAC em 2010. A pesquisa emprega o método de análise custo-benefício, onde o valor de custo ambiental ultrapassa 22 milhões de pesos, com maior incidência no financiamento para a sustentabilidade, seguido por perda de benefício econômico do sistema produtivo. O benefício econômico ambiental ultrapassa os 898 milhões de pesos. O maior percentual tem bens em conservação, com $45,48 \%$. A relação custo-benefício apresenta um resultado interessante com 39 pesos (benefício) para cada unidade de custo ambiental e um valor atual de 3.641 milhões. Os resultados, além de inovadores, contribuem para o processo de tomada de decisões para o desenvolvimento sustentável.

Palavras-chaves: Valoração econômica ambiental. Análise custo-benefício. Bens e serviços ambientais. Área costeira de Camagüey.

\footnotetext{
${ }^{1}$ Dra. en Ciencias Económicas. Centro de Investigaciones de Medio Ambiente de Camagüey, Cuba. E-mail: zeque@cimac.cu

${ }^{2}$ MSc en Administración de Empresas. Centro de Investigaciones de Medio Ambiente de Camagüey, Cuba. E-mail: ernesto.figueredo@cimac.cu

${ }^{3}$ Dr. Ciencias Económicas. Universidad de Camagüey, Cuba. E-mail: aristide.pelegrin@reduc.edu.cu

4 Dr. Ciencias Económicas. Dpto de Matemátcas, Universidad de Pinar del Río, Cuba. Bolsista de Posdoctorado de CAPES/Brasil. Programa de Pós-Graduação em Tecnologia (PPGTE/UTFPR) Email: santoyocuba@gmail.com

5 MSc en Administración de Empresas. Universidad de Camagüey, Cuba. E-mail: silvina.varona@reduc.edu.cu
} 


\begin{abstract}
In the northern coastal zone of Camagüey province, an important tourist development is being planning in the medium term. The study area consists of $4062,0 \mathrm{~km}^{2}$ (mainland, keys and marine area) and is limited to the level curve of 5 meters. It is a very fragile ecosystem because there are ten protected areas, one of them with international importance (Wildlife Refuge "Río Máximo" of Camagüey). The aim of this work is to analyze the cost-benefit ratio to the northern coastal zone of Camagüey province, Cuba. It's used a methodological procedure designed and validated in the Territorial Project "Analysis of the environmental goods and services production in the northern coastal area of Camaguey province, Cuba" executed by the CIMAC in 2010. The cost-benefit method is applied, which the environmental cost value exceeds 22 million with higher incidence in funding for sustainability, followed by the loss of economic benefit of the productive system. The environmental economic benefit exceeds the 898 million pesos. The higher percentage belongs to goods in conservation with $45,48 \%$. The benefit-cost ratio presents an interesting result with 39 pesos (benefit) for each unit of environmental cost and a current value of 3641 millions. The results, besides to be novel, contribute to the decision-making process for sustainable development.
\end{abstract}

Keywords: Environmental economic valuation. Cost-benefit analysis. Environmental goods and services. Coastal zone of Camagüey.

\title{
RESUMEN
}

En la zona costera norte de la provincia de Camagüey se planifica un importante desarrollo turístico en el mediano plazo. El área de estudio está compuesta por $4062,0 \mathrm{~km}^{2}$ (tierra firme, cayos y área marina) y se limita hasta la curva de nivel de 5 metros. Es un ecosistema ${ }^{6}$ muy frágil porque existen diez áreas protegidas, una de ellas con importancia internacional (Refugio de Fauna "Río Máximo" de Camagüey). El objetivo del trabajo es analizar la relación costo-beneficio para la zona costera norte en la provincia de Camagüey, Cuba. Se utiliza el procedimiento metodológico diseñado y validado en el Proyecto Territorial "Análisis de la producción de bienes y servicios ambientales en la zona costera norte de la provincia de Camagüey, Cuba" ejecutado por el CIMAC en el 2010. Se emplea el método de análisis costo-beneficio, cuyo valor de costo ambiental supera los 22 millones de pesos con mayor incidencia en el financiamiento para la sostenibilidad, seguida por la pérdida del beneficio económico del sistema productivo. El beneficio económico ambiental supera los 898 millones de pesos. El mayor porcentaje lo tienen los bienes en conservación con un 45,48 \%. La relación costo-beneficio exhibe un interesante resultado con 39 pesos (beneficio) por cada peso de costo ambiental y un valor actual de 3641 millones de pesos. Los resultados obtenidos, además de novedosos, contribuyen al proceso de toma de decisiones para el desarrollo sostenible.

Palabras claves: Valoración económica ambiental. Análisis costo-beneficio. Bienes y servicios ambientales. Zona costera de Camagüey.

\footnotetext{
${ }^{6}$ Conjunto de componentes vivos e inertes, compleja y estrechamente relacionados, que actúan como un todo específico y que constituyen los ambientes, naturales e intervenidos.
} 


\section{INTRODUCCIÓN}

La zona costera norte de la provincia de Camagüey la integran ecosistemas ricos en valores naturales como las áreas protegidas. Entre estas se ubica un sitio Ramsar de Cuba: Refugio de Fauna "Río Máximo". También se ubica MaternilloTortuguilla, La Alegría-Laguna Larga, Alturas de Guajaba, Punta del Este, Correa, Alto del Puerto, Silla de cayo Romano y Alto de Juan Sáez.

Esta área de estudio está considerada hasta la curva de nivel de 5 metros la cual posee un área de $4062,0 \mathrm{~km}^{2}$ y completada por cuatro municipios. Está compuesta por zonas de tierra firme, cayos y área marina. La primera posee una extensión de $952.75 \mathrm{~km}^{2}$, la segunda $1955 \mathrm{~km}^{2}$ y el resto a la última. Los usos de suelo por municipios son: Esmeralda: pastos, bosques y tierras ociosas; Sierra de Cubitas: caña, cultivos varios, cítricos, bosque y terrenos ociosos; Minas: bosque, caña, pastos, cítricos y cultivos varios; Nuevitas: bosque, pastos, cultivos varios, extracción de minerales e industrias. Los fundamentales organismos tenentes son: MINAGRI (empresas pecuarias, cooperativas, granjas estarles, empresa forestal, empresa de cítricos y Empresa para la Protección de la Flora y la Fauna) y MININT (granjas cañeras). En los cayos, los principales usos son bosques y maleza. Cuentan con excepcionales playas y paisajes naturales.

Se encuentran enclavadas diez comunidades: La Gloria, Puerto Piloto, Palma City, Gurugú, Mola, Nuevitas, San Agustín, Punta de Ganado, San Agustín y La Boca. La población supera los 44 mil habitantes y un dato interesante es que excepto San Agustín y Mola, el resto tiene tipo de asentamiento espontáneo. El objetivo de este trabajo consiste en analizar la relación costo-beneficio para la zona costera norte en la provincia de Camagüey, Cuba y su aporte al proceso de toma de decisiones para el desarrollo sostenible.

\section{METODOLOGÍA}

Esta sección comprende tres momentos fundamentales de este trabajo. Inicialmente, se presentan los fundamentos teóricos metodológicos de la 
investigación sobre los principales métodos de valoración económica asociados a los recursos naturales y posteriormente se detalla el procedimiento metodológico propuesto para analizar la relación costo-beneficio para la zona costera norte en la provincia de Camagüey.

\section{CONCEPTO DE VALOR ASOCIADO A UN ECOSISTEMA}

Los ecosistemas comprenden una compleja articulación de factores capaces de ofrecer recursos naturales y funciones ambientales que dan sustento a la vida (DE GROOT ET AL., 2002; BOVARNICK; ALPIZAR; SCHNELL, 2010). En tal sentido, la ciencia económica contemporánea precisa encontrar aportaciones que tributen hacia el entendimiento sobre la necesidad de actuaciones humanas más racionales.

En torno a este propósito, la idea de la cuantificación del valor de los servicios de los ecosistemas se ha tomado como un mecanismo importante para asegurar el reconocimiento y aceptación del manejo público de los ecosistemas y para influir, mediante esta información en las políticas de conservación de los mismos (ALDEN, 1997; VILLA ET AL., 2002; SARMIENTO, 2003).

Esto significa que la valoración económica de los bienes y servicios ambientales ha ocupado una atención especial en los últimos años, por cuanto, la inexistencia de mercados reales dificulta conocer el verdadero valor de las funciones ambientales, lo que conduce a entender que:

\footnotetext{
Valorar económicamente al ambiente supone el intento de asignar valores cuantitativos a los bienes y servicios proporcionados por los recursos ambientales, independientemente de la existencia de precios de mercado para los mismos. Esto quiere decir que la necesidad de la valoración excede largamente al trabajo que hace el mercado otorgando precios y asignando recursos dentro de la economía (TOMASINI, 2012, p. 1).
}

Un análisis de dicho planteamiento conduce a reflexionar que la valoración económica del conjunto de bienes y servicios ambientales asociados a estos ecosistemas constituye en sí misma una importante herramienta que aporta la ciencia económica al proceso de toma de decisiones, más allá de su mera interpretación cuantitativa. 
En otras palabras, es posible afirmar que la valoración económica de bienes y servicios ambientales:

[...] traduce el impacto ambiental en valores que pueden ser comparados e integrados con criterios económicos y financieros (costo-beneficio) para tomar decisiones acertadas, dejando menos espacio para juicios subjetivos. La valoración provee un veraz indicador de actuación económica que puede representar una guía para la definición de políticas públicas, aplicación de impuestos, la asignación de subsidios o la decisión de gastar en conservación de recursos o de mitigación del impacto ambiental requiere de una adecuada valorización de los costos y beneficios ambientales a alcanzar TOMASINI, 2012, p. 1).

En relación a este proceso de valoración, la ciencia económica ha desarrollado la Teoría del Valor Económico Total asociado a los espacios naturales, la cual incorpora en su valorización diferentes usos de un determinado recurso, e incluso, el no uso de dicho recurso. Estos valores pueden ser desagregados, tal como se propone a continuación (PEARCE; TUNNER, 1995; DOSI, 2001, SARMIENTO, 2003).

Valor de uso: Son aquellos que se derivan del empleo real de los recursos naturales y ambientales y de los beneficios que se obtienen de ellos.

Valor de uso directo: Representa el valor de producción o de consumo de los componentes o funciones de los ecosistemas.

Valor de uso indirecto: Representa el valor de las funciones ambientales que apoyan o protegen la actividad económica.

Valor de opción: Representa el valor del medio ambiente como un uso potencial frente al valor de uso en sí mismo.

Valor de no uso: Responde al valor que posee un recurso por su propia existencia, sin que ello condicione su uso.

Valor e existencia: Representa la medida en que la sociedad está dispuesta a pagar para conservar recursos por sí mismos, con independencia de sus usos para la producción o el consumo.

Valor de legado: Representa la disposición a pagar para que las generaciones futuras puedan hacer uso de esos recursos. 


\section{MÉTODOS DE VALORACIÓN ECONÓMICA AMBIENTAL}

Ante el reto que representa la valoración económica de bienes y servicios ambientales, la economía ambiental ha diseñado instrumentos que permiten abordar estas cuestiones desde una perspectiva económica.

En este sentido, los criterios ofrecidos por Lindhjem et al. (2007); Winkler (2006), sostienen que los métodos de valoración económica enfatizan su análisis en las preferencias del consumidor, por lo cual, es preciso señalar que:

[...] mediante el uso de estos métodos es posible obtener información que permite ampliar las magnitudes macroeconómicas del sector proveniente de los recursos naturales como puede ser el sector turístico, forestal, agrícola, minero, pesquero, etc. (SARMIENTO, 2003, p.22).

Asimismo, García; Colina (2004) señalan el reto que ha representado para la ciencia económica la no existencia de mercados reales, al expresar que:

[...] la no existencia de mercados reales ha obligado a buscar técnicas de valoración económica que permitan deducir el citado valor a partir de la observación de mercados reales relacionados con el recurso natural (métodos indirectos o de preferencias reveladas) o a través de la obtención directa de las preferencias personales (métodos directos o de preferencias declaradas), (GARCÍA; COLINA, 2004, p.2).

Los métodos de valoración económica más empleados, se clasifican de acuerdo al origen y disponibilidad de la información en: (DIXON; HUFSCHMIDT, 1988; BARSEV, 2002, p.13).

- Métodos de valoración directos.

- Métodos de valoración indirectos.

- Métodos de valoración contingentes.

En la actualidad, las discusiones teóricas sobre la clasificación de estos métodos apuntan hacia la consideración de los métodos de valoración contingente como método directo (HERRUZO, 2002; CERDA, 2003; GARCÍA; COLINA, 2004) y la utilización generalizada de una nueva clasificación, asociada a la forma en la cual se obtienen las preferencias del consumidor, entiéndase, métodos de preferencias declaradas (directos) y métodos de preferencias reveladas (indirectos).

Entre los métodos de valoración económica ambiental más empleados, se encuentra: el método de valoración contingente (MVC), método del coste del viaje (MCV), método de precios hedónicos (MPH), método de costes evitados (MCE), modelos de elección (choice modelling) y otros que están fundamentados en la 
variación de los valores de la producción, valores de costes, indicadores medioambientales y en razonamientos matemáticos complejos. Los mismos están basados en la medida de la disposición a pagar (DAP) o en la disposición a ser compensado (DAC), mediante la creación de mercados hipotéticos o directamente apoyándose en mercados reales y teniendo sesgos propios de las personas. (SARMIENTO, 2003 p.3)

La utilización de estos métodos se encuentra estrechamente vinculada a la valoración de recursos naturales, específicamente al valor económico asociado a un determinado ecosistema. En este caso, se destaca la utilización del análisis costobeneficio $(A B C)$, extendido como un mecanismo práctico en la evaluación de proyectos de inversión (VREEKER ET AL., 2001; SARMIENTO, 2003).

La aplicación del ACB es muy útil cuando la finalidad es decidir si realizar una inversión o no, pues comprende el análisis y comparación de los beneficios que se espera recibir y los costos que se estima serán necesario incurrir, (MOONS, 2002).

\footnotetext{
Este método presenta como ventaja que la mayoría de los beneficios y costos pueden ser empleados de diversas maneras brindando mucha información. El valor que encierra el (VAN), representa mucha información al igual que la tasa interna de retorno (TIR), o el de la relación de costo de beneficio (RCB) (SARMIENTO, 2003, p.14).
}

\section{PROCEDIMIENTO PROPUESTO}

A continuación, se definen los componentes que comprenden los beneficios económicos, los costos ambientales y el análisis costo-beneficio asociados a la zona norte costera de la provincia de Camagüey.

\subsection{BENEFICIOS ECONÓMICOS}

En el estimado económico de los beneficios ambientales (o ecosistémicos) se utilizan los respectivos términos valores de uso directo, indirecto y de opción.

Valor de uso directo: Representa la suma de los indicadores (producto de la cantidad por el precio) asociados a cada elemento de la diversidad biológica, en 
correspondencia a su uso y manejo. Incluye los bienes comercializados y de uso consuntivo ${ }^{7}$, tasados a sus correspondientes precios locales de mercado.

Para estimar los bienes económicos del sector estatal, se maneja la producción mercantil de la entidad correspondiente ${ }^{8}$ en tanto que, para el sector no estatal se acepta el indicador ingreso, el cual coincide con venta, debido a las particularidades de esta actividad.

Los estimados para el sector estatal en la zona de estudio, se corresponden con el promedio de seis años ordenados por sectores económicos del sistema productivo. La tierra firme del área del proyecto realiza importantes aportes a la economía. En el monto total del beneficio económico, cuenta significativamente el municipio Nuevitas (con fuerte actividad industrial: producción de energía ecléctica, fertilizantes, pintura, cemento, alambre de púa, lácteos, y turismo).

La pesca privada resulta de interés particular para la protección ambiental, pues en los talleres realizados los días de expediciones y trabajos de campo, se pudo apreciar que esta actividad es significativa ${ }^{9}$. Se realiza el cálculo de esta actividad económica, en todas las comunidades ubicadas en la zona costera hasta la cota de 5 metros, con la participación de agentes externos y la colaboración de algunos pescadores y familiares. Se enriquece la búsqueda de datos, a partir de informaciones ofrecidas por empleados por la conservación ubicados en los cayos, pescadores dedicados a la actividad de pesca flay y con trabajadores ejerciendo la actividad, en la plataforma del territorio con barcos que corresponden a otros territorios del país. Finalmente se estima el valor de uso directo total del área del proyecto y su rendimiento productivo ${ }^{10}$.

Valor de uso indirecto: Beneficio económico prestado por concepto de valor de uso indirecto en la zona costera norte de Camagüey, significa un ahorro para la sociedad cubana. Se agrupan en tres variables: captura de $\mathrm{CO}_{2}$, oferta de agua, y control de erosión y formación del suelo.

\footnotetext{
${ }^{7}$ Productos de la naturaleza que son consumidos directamente, sin pasar por un mercado. Al valor del uso consuntivo se le puede asignar el precio del mercado local (donde es consumido).

${ }^{8}$ Significa la expresión en valor, a precios de empresa, de los bienes, trabajo y servicios terminados y/o producidos en el período, los cuales se informan y se destinan a la venta.

${ }^{9}$ Es obvio que esta requiere atención especial dentro del manejo integrado, así como, en el programa de educación ambiental con vistas a favorecer una captura sostenible, manejando un arte de pesca armonioso con el ecosistema. Para tales fines se ejecuta con rigor un muestreo de forma participativa y anónima, para aproximarse a la realidad del beneficio económico que aporta la diversidad biológica en cuestión, por este concepto.

${ }^{10}$ Relación entre bienes producidos para uso directo y consuntivo y el total del área de estudio.
} 
- Captura de $\mathrm{CO}_{2}$ : Para la valuación de este servicio ambiental ${ }^{11}$ se utiliza la capacidad de captura para bosque tropical ${ }^{12}$ y manglar ${ }^{13}$. Los resultados se alcanzan empleando el SIG.

- Oferta de agua: Se realiza el estimado bajo el planteamiento hipotético de interrupción del servicio natural por pérdida de las funciones ecosistémicas inmediatas $^{14}$. En tal caso, la cuña salina avanzaría tierra adentro ${ }^{15}$, por tanto, el efecto de la concentración aumentaría y por ende, la migración de los iones debido al movimiento por la diferencia del gradiente de concentración ${ }^{16}$.

La tasación se realiza a partir del gasto evitado por concepto de inversión hidráulica ${ }^{17}$ :

- Distancias del proyecto sustituto: en este caso, se maneja la distancia entre el reservorio alternativo (nueva fuente de abasto propuesta por el Instituto Nacional de Recursos Hidráulicos en la Provincia) y las respectivas comunidades de pobladores ${ }^{18}$. Se utiliza el SIG.

- Precio unitario del de proyecto sustituto: promedio del costo estimado de la inversión hidráulica por km, propuesto por la Empresa de Investigaciones de Proyectos Hidráulicos de Camagüey (23 440 pesos/Km. anuales).

${ }^{11}$ Con la aprobación del Protocolo de Kyoto (1997), los recursos boscosos se vuelven canjeables como sumideros de carbono en beneficio de países industrializados.

${ }^{12}$ Existen varios estimados de captura de $\mathrm{CO}_{2}$ en el mundo para bosques tropicales. En este trabajo se utiliza 81.25 ton/ha obtenido por Benítez en "Estimación de la biomasa total en plantaciones de casuarina equisepitolia FORTT en la provincia de Camagüey". Universidad de Alicante. 2006.

${ }^{13}$ Se transfiere el beneficio económico de captura de $\mathrm{CO}_{2}(103.5$ ton/ha) para este tipo de bosque en el ecosistema Sabana Camagüey. Gómez, (ob.cit.). porque pertenece a la misma área objeto de estudio de esta autora.

${ }^{14}$ Funciones ecosistémicas inmediatas: Incluyen todas las relaciones entre el sistema boscoso y la cuenca mayor, donde sus impactos positivos o negativos tienen significación.

${ }^{15}$ Este desplazamiento está en dependencia también de otros factores combinados como el relieve, la actividad económica humana y la entrega de agua dulce (tierra-mar), entre otros; pero en este análisis se asume que éstos se mantienen constantes.

${ }^{16}$ Bajo esta simulación, los asentamientos poblacionales, así como los tenentes agrícolas de la zona de estudio, se verán afectados por la insuficiente calidad del líquido, la disminución del rendimiento de las cosechas u obligados a desestimar los tradicionales usos del suelo. Entonces, el Estado estaría obligado a sustituir el servicio con la inversión de ingeniería.

${ }^{17}$ La salinidad es un elemento de importancia para la calidad del agua destinada al consumo humano por cuanto, una variación en su composición química provoca diferentes enfermedades como las cardiovasculares, renales y de hipertensión arterial (HTA), entre otras. La Norma Cubana 93- 02 de 1985, señala que el agua potable tiene que cumplir con los requisitos físicos, químicos y microbiológicos establecidos.

${ }^{18}$ El propio informe contiene las distancias medidas en línea recta hasta la zona de la fuente más conveniente, generalmente todos los pozos que tienen estas comunidades hoy día, están fuera de norma la bacteriología (Colis fecales y totales) que con cloración se resuelve. 
- Control de erosión y formación del suelo ${ }^{19}$ : Se continúa con la situación hipotética anterior, referida en este caso, al impacto ambiental ${ }^{20}$ para la calidad de los suelos ${ }^{21}$ asociada a la salinización ${ }^{22}$. Para mejorar la calidad de un suelo salinizado, el proceso recurrente en la práctica es la utilización de fertilizantes, preferentemente naturales, por ejemplo el compost ${ }^{23}$, que brinda beneficios tanto productivos como ecológicos.

Se asumen los gastos evitados por fertilización el suelo para mantener sus condiciones naturales. Se acepta que para mejorar las condiciones del suelo salinizado se requieren $45 \mathrm{t}$ de compost por $1 \mathrm{ha}^{24}$ mientras que la restauración de 1 ha de suelo salinizado se estima entre 9000 y 27000 pesos $^{25}$. En este último se trabaja con el costo mínimo. En ambos casos se utiliza el SIG.

Valor de opción: Se distinguen dos variantes: usar (comercializar o consumir) y uso con manejo. Se operan con las variables vegetación, fauna y agua:

- Vegetación: Formación boscosa ubicadas en la zona de tierra firme y cayos del ecosistema objeto de estudio. Se estima el costo de oportunidad por tipo

${ }^{19}$ El incremento de las áreas con baja fertilidad es el resultado de varios factores, entre los que se encuentran los procesos erosivos, que han eliminado la capa más fértil del suelo, y la mala utilización de tecnologías de cultivos, que provocan su degradación química, física y biológica.

La desertificación, por su parte, es el resultado del desarrollo y la combinación de los procesos degradativos antes mencionados. Fundamentalmente, la erosión, la salinidad y la eliminación de la vegetación, que induce cambios condicionantes hacia un decrecimiento de las regiones pluviométricas y del potencial biológico del suelo.

Existen otras acciones degradativas no contempladas como factor limitante, tales como la minería a cielo abierto y las zonas de préstamo, que aunque actúan en menor área, no son despreciables para el país, por su efecto devastador.

${ }^{20}$ Cualquier cambio neto, positivo o negativo, que provoca sobre el ambiente como consecuencia indirecta, de acciones antrópicas susceptibles de producir alteraciones que afecten la salud, la capacidad productiva de los recursos naturales y los procesos ecológicos esenciales.

${ }^{21}$ El suelo tiene características particulares como la fertilidad, es decir, la facultad de satisfacer las necesidades de las plantas con elementos nutritivos y agua, asegurar sus sistemas radiculares y brinda una cantidad de aire y calor para su desarrollo normal por tanto, asegura en gran medida las producciones agrícolas, constituyendo esta una razón fundamental para su protección.

${ }^{22}$ La calidad del suelo también está en dependencia del tipo de éste: pardo, carbonato, salino o de otro tipo. Para evaluar y clasificar un suelo por su productividad resulta fundamental conocer los factores limitantes (son aquellos que provocan reducción en las posibilidades de su uso agrícola) y sus interacciones.

${ }^{23}$ Fertilizante que se aplica para el mejoramiento del suelo a partir de componentes naturales. Existen otros tipos como son los residuos de cosechas y el estiércol, que constituyen fertilizantes procesados así como la materia orgánica que es un fertilizante no procesado.

${ }^{24}$ Datos suministrado por el Instituto de Suelos Camagüey.

${ }^{25}$ lbíd. 
de formación boscosa, la biomasa estimada y el precio del productor o tenente para madera, leña y saco de carbón ${ }^{26}$ :

> Bosque: Bosques Conocarpus erectus, Bosque Siempreverde Micrófilo, Bosque semideciduo mesófilo, Bosque Siempreverde de galería, Bosque Siempreverde de ciénaga y Bosque semideciduo micrófilo. Posee un área total de 173550.4 ha de éstas, el 50\% respectivamente corresponde a tierra firme y cayos. Se identifican las alternativas siguientes: costo de oportunidad de madera ${ }^{27}$ y costo de oportunidad de leña ${ }^{28}$. La cantidad de metros cúbicos de madera se obtiene por el total de madera en pie que (126.1 millones de metros cúbicos) y las hectáreas de bosques que existen en el país (2434.98 miles de hectáreas) ${ }^{29}$. Una alternativa es la leña para combustible. Se calcula la biomasa sobre la base del $65 \%$ de los metros cúbicos de madera ${ }^{30}$. Se tasa la biomasa calculada ${ }^{31}$ a 6.01 pesos el $\mathrm{m}^{3}$. Con el SIG estos datos se extrapolan al área de estudio, se estima la biomasa y se realiza la tasación correspondiente.

> Mangle: Bosque Siempreverde Manglar Mixto de Rhyzophora Mangle A.germinans, Bosque Siempreverde de Rhyzophora Mangle A.germinans Lr, Conc, Manglar de Rhyzophora Mangle. Se dispone de datos sobre la producción de madera del manglar y se obtiene la producción potencial para esta formación boscosa. No se desecha la posibilidad de que el manglar sea manejado para la extracción de madera $^{32}$. En tal alternativa, la explotación del recurso debe tener en

\footnotetext{
${ }^{26}$ El precio del productor utilizado es el registrado en la Empresa Forestal Provincial. Sus distorsiones con relación al precio económico no son significativas.

${ }^{27}$ El uso de la madera constituye un recurso productivo importante para la economía nacional. Entre otros, postes de tendido eléctrico y telegráfico, traviesas, paletas, cujes para tabaco, cajas, módulos de envase y otros.

${ }_{28} \mathrm{El}$ uso de los bosques como energía tiene importancia para la economía familiar (rural fundamentalmente) y territorial (exportación de carbón).

${ }^{29}$ Colectivo de autores. Manejo Forestal. Módulo de Formación Básica, Proyecto: Acciones prioritarias para consolidar la protección de la Biodiversidad en el ecosistema Sabana Camagüey. (s.p.d.i).

${ }^{30}$ lbíd.

${ }^{31}$ Empresa Forestal Provincial. Camagüey.

${ }^{32}$ Habitad de cría natural de numerosas especies de peces, crustáceos y moluscos, y refugio de una rica fauna endémica y migratoria. Son fuertemente impactados negativamente por fenómenos naturales o antrópicas, que en muchos casos pueden ser atenuadas o evitadas, con la aplicación de manejos adecuados, para que su uso sea racional. La biomasa arbórea debe ser manejada como elemento indispensable para rehabilitar y conservar el ambiente y prevenir el desarrollo de los procesos conducentes a la desertificación y la sequía.
} 
cuenta el incremento medio anual del mismo (IMA: 4, $2 \mathrm{~m}^{3} / \mathrm{ha} / \mathrm{año}$ ), lo cual significa que no se debe extraerse una cantidad superior al promedio anual de recuperación natural del bosque. Los autores comparten y resaltan el respeto a este principio, por lo que el estimado se corresponde con la producción potencial anual.

Otra alternativa para el costo de oportunidad sería la producción de carbón ${ }^{33}$. Con una disponibilidad de $20 \mathrm{~m}^{3}$ de cuerda de leña se producen $30 \mathrm{sacos}^{34}$. Estos datos se extrapolan al área de estudio usando el SIG para obtener la biomasa correspondiente y se ejecuta la tasación.

- Fauna: Contempla el inventario en poder del tenente para Río Máximo y se incluye además, otros recursos con igual tratamiento, como los huevos (flamenco, cocodrilo, etc.) entre otros. Para el resto de las especies, se usa el inventario bajo el mismo esquema ${ }^{35}$. También se estima la biomasa del ganado reproductor vacuno, equino, ovino, caprino, porcino, avícola y búfalo ${ }^{36}$ y se tasa por tipo con el precio correspondiente del productor ${ }^{37}$.

Según el listado de especies en conservación del tenente Empresa Nacional de Flora y Fauna en Camagüey, existen abundantes individuos, muchas de ellas exóticas y carismáticas. En el momento del levantamiento de esta información, la entidad mencionada anteriormente, no contaba con la totalidad de los inventarios por lo que no es posible evaluar al universo.

En el Refugio de Fauna "Río Máximo", sitio de interés internacional con importantes valores naturales se identifican 20000 ejemplares de aves marinas ${ }^{38}$. También se reflexiona, que otras especies de aves estarían limitadas por el tipo de alimentación y la adaptación en cautiverio. Por ejemplo el pelícano consume pescado lo que provocaría que su alimentación en cautiverio fuera muy costosa. En el caso del delfín se trata de una especie que posee demanda en el mercado

\footnotetext{
${ }^{33}$ Debido a la calidad que históricamente tiene el carbón de mangle, este producto está demandado en el mercado local e internacional) por lo que constituye la mejor alternativa económica.

${ }^{34}$ Empresa Provincial Forestal. Camagüey.

${ }^{35}$ Precios identificados en la Empresa Nacional de Flora y Fauna de Camagüey.

${ }^{36}$ No se incorpora la masa total por cuanto, a criterio de los autores, las reproductoras constituyen la base del sostenimiento de la población de estas especies. Además, está presente la necesidad de minimizar sesgos que pueden estar introducidos por las edades y desarrollo de las respectivas especies ya que en la conversión se utiliza la media ponderada de unidades (cabezas) a kilogramos de biomasa.

${ }^{37}$ Empresa de Aseguramiento y Servicio. Ministerio de la Agricultura. Camagüey.

${ }^{38}$ Ficha de los humedales de Ramsar. CENAP.
} 
internacional para las actividades extrahoteleras. En el estimado se consideran las alternativas siguientes: costo de oportunidad de los flamencos, costo de oportunidad de aves acuáticas, costo de oportunidad del cocodrilo, costo de oportunidad de los delfines, costo de oportunidad del venado y costo de oportunidad de la jutía.

- Agua: Para este costo de oportunidad se usa el balance hídrico por cuencas ${ }^{39}$ el cual permite conocer un aproximado de la disponibilidad de agua. Se debe garantizar el gasto ecológico para proporcionar bienes y servicios por lo que, se hace indispensable respetar esta demanda e identificar las alternativas de usos del agua. El cultivo de arroz es una de las producciones con mayores demandas del líquido en la actividad agrícola. Este renglón productivo consume por ciclo 3072000 metros cúbicos de agua en la zona de explotación ${ }^{40}$.

Como mejor alternativa se selecciona el uso agrícola (clasificación para América) y se multiplica por el $70 \%$ la disponibilidad de agua en la zona de estudio ${ }^{41}$, la cantidad del uso del agua para la mejor variante señalada a inicios del párrafo (74\%) y el precio del líquido ${ }^{42}$.

\subsection{COSTOS AMBIENTALES}

En este documento se enfoca al costo medioambiental como el monto estimado por concepto de gastos fijos y variables para manejar, investigar o administrar con el objetivo de mitigar un daño real o potencial ocasionado por el subsistema económico-social. Representa el monto de recursos financieros, materiales y humanos que el Estado y/o entidades estatales y no estatales debe planificar en su presupuesto con fines de prevenir, mitigar o restaurar una

\footnotetext{
${ }^{39}$ Se realiza con fines para el presente trabajo por lo que se reconocen algunas particularidades. En este caso falta determinar aproximadamente el volumen que se infiltra, además precisar todas las corrientes superficiales y cuerpos de agua que evaporan. En general, el sesgo por estos conceptos declarados anteriormente en el resultado final del área (contiene volúmenes que constituyen pérdidas en el balance) no impide la valoración económica del recurso a través del costo de oportunidad.

${ }^{40}$ La cosecha del arroz tiene dos ciclos en el año. La primera se efectúa en el mes de octubre y la segunda se realiza en la primavera con un consumo promedio por hectáreas de 20000 metros cúbicos en cada ciclo de cosecha.

${ }^{41}$ Entrega máxima y se convierte la disponibilidad de $\mathrm{hm}^{3} \mathrm{a} \mathrm{m}^{3}$.

${ }^{42}$ El precio comercialización del agua en Cuba es de 0.30 centavos por $\mathrm{m}^{3}$.
} 
externalidad negativa ${ }^{43}$. Incluye además, la pérdida total o parcial de los bienes resultados de la materialización del trabajo abstracto ${ }^{44}$ así como los productos y funciones medioambientales dañados o sin aprovechamiento económico.

En la literatura revisada se manejan varias clasificaciones o tipos de costos ambientales. En este trabajo se respeta estas interesantes clasificaciones pero, se requiere de una nueva propuesta la cual se fundamenta a continuación.

Cuando se agrega valores en el sistema productivo y la mercancía no se realiza, según el destino para el cual está planificada, entonces se puede producir una pérdida total o parcial para el productor pero también para el medio ambiente y la sociedad. Aún cuando el producto pueda tener otro aprovechamiento económico (reciclado), lo cierto es que existe, además del gasto de trabajo social, un determinado consumo de energía y de recurso natural. En este caso, existe un ingreso o beneficio económico social y ambiental perdido por tanto, se debe considerar como un costo ambiental por lo que se incorpora a la ganadería (concepto de muertes) del área de estudio.

La actividad económica genera impactos negativos al medio ambiente a través de la contaminación. Las entidades deben contar con sistemas de gestión y manejo de desechos o al menos elementales acciones preventivas, correctivas o de mitigación. La implementación de éstas significa un costo para el productor el cual, debe estar debidamente planificado en el presupuesto de cualquier forma de propiedad.

Para el cumplimiento de sus responsabilidades y misiones, la provincia de Camagüey cuenta con una estructura institucional y organizacional que permite fortalecer el manejo, la investigación de la capacidad de carga del ecosistema y otros intereses en la zona costera norte. La delegación provincial del Ministerio de Ciencia, Tecnología y Medio Ambiente cuenta con un grupo de administración, gestión e inspección ambiental respectivamente. De forma articulada y cooperada, existen entidades estatales territoriales para la investigación y/o acciones dirigidas al manejo del medio ambiente. Esta infraestructura e investigaciones son costosas.

\footnotetext{
${ }^{43}$ Efectos peligrosos del proceso económico que se generan por no asumir éste todos los costos de su actividad productiva. Los costos sociales son la suma de los costos de estas externalidades y los recursos naturales a los que se les ha asignado un precio.

${ }^{44}$ Trabajo social gastado en su producción.
} 
Por otra parte, las entidades, estatales y no estatales, tienen la obligación de usar tecnología y técnicas armoniosas con el medio ambiente; de restaurar daños ocasionados y de mitigar los impactos negativos a través de inversiones (en correspondencia con el desarrollo económico).

En opinión de los autores de este trabajo, la clasificación de los costos ambientales debe estar en función de los objetivos del proyecto de investigación, el estado y disponibilidad de las informaciones del sistema estadístico y la dimensión y alcance de los resultados (salidas). Por tanto, para favorecer a los objetivos y estructura de la planificación, los programas de planes de manejos y planes operativos en áreas protegidas así como el manejo integrado de la zona costera y la política general de Cuba referida a la gestión y control del medio ambiente; se utilizan las siguientes categorías:

a) Sostenibilidad: manejo ${ }^{45}$ (inversión por prevención, mitigación o restauración de daños, monitoreo ${ }^{46}$, educación ambiental ${ }^{47}$, vigilancia $^{48}$ y gestión ambiental ${ }^{49}$ ), investigación ${ }^{50}$ y administración ${ }^{51}$.

b) Pérdida del beneficio económico de la producción de bienes materiales.

c) Financiamiento de medidas de mitigación, preventivas o correctivas a cargo de los contaminadores.

\footnotetext{
${ }^{45}$ Acción planeada para hacer evolucionar un sistema, de modo tal, que se puede derivar el mejor provecho de este a corto plazo y preservarlo para su utilización a largo plazo. Una forma o tipo de manejo puede ser deseable para determinados usos, pero inconveniente para otros. Para lograr un adecuado manejo, es necesario evaluar las ventajas y desventajas de las alternativas pero sin obviar la conservación. Contiene el monto de inversión y operación con fines de prevención, mitigación o restauración.

46 Proceso de observación repetitiva, con objetivos bien definidos relacionado con uno o más elementos del ambiente, de acuerdo con un plan temporal.

${ }^{47}$ Proceso educativo mediante el cual el educando adquiere la percepción global y pormenorizada de todos los componentes del ambiente, tanto natural como social, de la interdependencia y el funcionamiento de los ecosistemas, de la necesidad de su preservación y de su compatibilidad con el desarrollo.

${ }^{48}$ Sistema técnico, organizado para obtener datos periódicos de la contaminación existente en determinada zona.

${ }^{49}$ Conjunto de procedimientos mediante los cuales una entidad puede intervenir para modificar, influir u orientar los usos del ambiente así como los impactos de las actividades humanas sobre el mismo.

${ }^{50}$ Conjunto de proyectos de I+D territoriales, ramales y nacionales para contribuir con el desarrollo sostenible.

${ }_{51}$ Fuerza de trabajo contratada para control del recurso financiero y material con facultad de reglamentar, controlar y optimizar su uso. Incluye los gastos por concepto de logística y materiales para cumplimentar las acciones y tareas de la gestión ambiental así como los gastos generales que ocasiona la inspección ambiental.
} 


\subsection{ANÁLISIS COSTO-BENEFICIO}

En la evaluación del beneficio costo se emplean las técnicas tradicionales de evaluación de proyectos donde se identifican como ingreso a los beneficios ambientales y como egresos a los costos ambientales. El resultado muestra el beneficio ambiental por peso del costo ambiental actualizado para resaltar la importancia económica ambiental de esta zona $\operatorname{costera}^{52}$. Las premisas generales son las siguientes:

- El período analizado está comprendido entre el 2016 al 2022.

- Es válido utilizar indicadores estáticos.

- Los criterios de los expertos son decisivos.

- La tasa de actualización será del $8 \%$.

- Se trabaja la depreciación por el método lineal al $4 \%$ del activo fijo tangible.

- Para la evaluación con proyecto se declaran como ingresos a los bienes y servicios ambientales (valor de usos directo como reales, indirecto y de opción como potenciales).

- Se consideran en los egresos a los costos ambientales cargados por el manejo, la investigación, la administración y la implementación de medidas de mitigación.

- Se trabaja con moneda total.

\section{RESULTADOS}

\subsection{BENEFICIOS ECONÓMICOS}

A partir de las categorías declaradas en la concepción metodológica, se obtiene una primera aproximación de los bienes y servicios ambientales por tanto, el monto está integrado por los estimados del valor de uso directo e indirecto así como

\footnotetext{
52 Los resultados obtenidos constituyen una primera aproximación para el tópico tratado. Los resultados son interesantes además, para la educación económica y ambiental de pobladores, directivos y tomadores de decisiones con vistas a concretar proyectos de desarrollos sostenibles. El beneficio económico perdido en la base productiva está subvalorado por no incluir todos los renglones productivos del sistema local.
} 
los de opción contenida en los respectivos artículos del propio compendio de resultados. A continuación se exponen los aspectos más sobresalientes en cada una de las categorías:

Tabla 1. Estimado total del beneficio económico ambiental, estructura porcentual y su rendimiento en la zona costera norte de Camagüey.

\begin{tabular}{|l|c|c|c|}
\hline $\begin{array}{c}\text { Categoría Económica } \\
\text { Ambiental }\end{array}$ & $\begin{array}{c}\text { Beneficios } \\
\text { Económicos } \\
\text { Ambientales } \\
\text { (MP) }\end{array}$ & $\begin{array}{c}\text { Estructura } \\
\text { Porcentual (\%) }\end{array}$ & $\begin{array}{c}\text { Rendimiento } \\
\text { Económico y } \\
\text { Ambiental } \\
\text { (MP/Km } \mathbf{2}^{\mathbf{5}}\end{array}$ \\
\hline Total valor de uso directo & 181020 & 20,15 & 44,6 \\
\hline $\begin{array}{l}\text { Total valor de uso } \\
\text { indirecto }\end{array}$ & 308663 & 34,37 & 76,0 \\
\hline Total valor de opción & 408462 & 45,48 & 100,6 \\
\hline \multicolumn{1}{|c|}{ Total } & $\mathbf{8 9 8 1 4 5}$ & $\mathbf{1 0 0 , 0}$ & $\mathbf{2 2 1 , 1}$ \\
\hline
\end{tabular}

Fuente: Elaborada por los autores

Valor de uso directo: El sector estatal representa el $66,4 \%$ de beneficio económico con mayor expresión en Nuevitas, seguido por Esmeralda. La industria es la predominante $(86,7 \%)$ seguido por el agropecuario (9,3\%). El turismo es moderado pero tiene un potencial de explotación significativo para el país y en particular para la provincia.

Valor de uso indirecto: En este resultado el mayor valor le corresponde al recurso natural suelo con el $68 \%$ en la estructura, aspecto que resulta muy revelador para el manejo sostenible de los suelos.

Valor de opción: El agua representa el mayor ingreso potencial y rendimiento ambiental por lo que, desde el punto de vista económico ambiental, el resultado obtenido se convierte en el aspecto de mayor interés para el manejo integrado de la zona costera.

\subsection{COSTOS AMBIENTALES}

\footnotetext{
${ }^{53}$ Significa la expresión del valor de uso directo, indirecto y de opción por $\mathrm{km}^{2}$. del ecosistema en general (4 062,0 $\mathrm{km}^{2}$ de zona terrestre firme, emergida y marina), por cuanto el mismo funciona como un todo a partir de la interacción de sus elementos de la biodiversidad.
} 
El $42 \%$ es por concepto de pérdida del beneficio económico, $44,6 \%$ por financiamiento para la sostenibilidad y el $13,3 \%$ por medidas de mitigación para los contaminadores en la zona costera norte de Camagüey ${ }^{54}$.

Tabla 2. Cálculo del costo ambiental por conceptos de sostenibilidad y pérdida del beneficio económico para la zona costera norte de la provincia de Camagüey, Cuba. (MP).

\begin{tabular}{|c|r|}
\hline Clasificación del costo ambiental & Estimado total \\
\hline Por concepto de sostenibilidad & 13190,9 \\
\hline$\bullet \quad$ De manejo & 6367,5 \\
\hline - De investigación & 384,3 \\
\hline - De administración & 3415,0 \\
\hline$\bullet \quad$ Medidas de mitigación & 3024,1 \\
\hline Por concepto de pérdida del beneficio económico & 9605,8 \\
\hline TOTAL & $\mathbf{2 2 ~ 7 9 6 , 7}$ \\
\hline
\end{tabular}

Fuente: Elaborada por los autores

El costo por concepto de sostenibilidad es el de mayor significación. Su estimado asciende a más de 10 millones de pesos al año. En la categoría de manejo están incluidos los costos actuales por mantenimiento de lagunas de oxidación atendidas por el presupuesto municipal (4) y gastos incurridos por la Empresa Nacional de Flora y Fauna de Camagüey así como por las oficinas territoriales del CITMA en los cuatro municipios costeros.

Se identifica el costo ambiental por investigación, expresión del presupuesto gastado en proyectos de investigaciones nacional, ramal y territorial (5) y por concepto de servicio (1). Seguidamente se muestran los costos ambientales por administración el cual contempla los gastos directos e indirectos de las cuatro oficinas territoriales del CITMA y de la Empresa Nacional de Flora y Fauna de Camagüey. Finalmente, se adiciona el costo ambiental por concepto de pérdida de beneficio económico con un monto de más de 9 millones de pesos en toda el área de estudio.

Del cálculo total, la Empresa Nacional de Flora y Fauna de Camagüey ejecuta más de cuatro millones de pesos por concepto de gasto para fines de conservación en todas sus áreas protegidas ubicadas en la zona de estudio. Los

\footnotetext{
${ }^{54}$ En particular, la contaminación en la Bahía de Nuevitas, territorio donde se desarrollan múltiples producciones industriales, constituye una importante amenaza para la zona costera porque en el mediano plazo pudiera modificar el paisaje del ecosistema donde se planifica un desarrollo turístico en la cayería. En tal sentido, se identifican medidas acompañadas con inversiones ambientales para mitigar el impacto negativo las cuales significan un costo.
} 
programas de manejo para la conservación así como vigilancia y protección, constituyen el $56 \%$ del monto total ejecutado.

La implementación de las medidas de mitigación, producto de la presión ambiental creada por la actividad económica, ocasiona un costo para los contaminadores. Su estimado constituye un indicador importante el cual debe ser considerado en el flujo de caja realizado en la última etapa del esquema metodológico de la investigación.

El total de medidas de mitigación (37) para disminuir los problemas ambientales identificados en la zona costera alcanzan un estimado superior a los tres millones de pesos; de estos el 54,9\% corresponden a Nuevitas. Por tanto, significaría una contribución que los contaminadores deben asumir en sus respectivos presupuestos en cumplimiento de su responsabilidad ambiental y social.

\subsection{ANÁLISIS BENEFICIO COSTO}

Los recursos biológicos ${ }^{55}$ considerados propician beneficios económicos. Sin embargo, estos pueden decrecer en el tiempo si los productores de los municipios costeros de referencia no incrementan su cultura económica ambiental. Es necesario dirigir la misión de sus organizaciones económicas hacia una producción más limpia ${ }^{56}$, en la medida en que el presupuesto para el desarrollo así lo permita (recuperación de la economía); sobre la plataforma de un adecuado sistema de gestión ambiental ${ }^{57}$.

El valor actual de los beneficios ambientales estimados en el ecosistema supera los 3641 millones de pesos y representan 39,4 pesos actualizado por cada peso de costo ambiental actualizado. En este trabajo se evidencia, además, la importancia del mecanismo de cobro y pago por servicio ambiental para el uso de un ecosistema, en este caso la cayería norte de la provincia de Camagüey.

\footnotetext{
${ }^{55}$ Son aquellos componentes de la biodiversidad que admiten un uso directo, indirecto o potencial.

${ }^{56} \mathrm{La}$ aplicación continúa de una estrategia integrada de prevención ambiental a los procesos y a los productos, con el fin de reducir los riesgos a los seres humanos y al medio ambiente.

57 Aquellos aspectos de los sistemas generales de una empresa, incluyendo las organizaciones, prácticas y recursos, que llevan a cabo y dan apoyo a la función de gestión ambiental.
} 


\section{CONCLUSIONES}

La investigación presentada, constituye un importante resultado del Proyecto Territorial "Análisis de la producción de bienes y servicios ambientales en la zona costera norte de la provincia de Camagüey, Cuba", que coordina el Centro de Investigaciones de Medio Ambiente de Medio Ambiente de Camagüey (CIMAC). Una vez realizada la misma, fue posible dar cumplimiento al objetivo propuesto, dirigido a analizar la relación costo-beneficio asociada al conjunto de bienes y servicios ambientales definidos para el área objeto de estudio.

Los resultados obtenidos en este trabajo son interesantes para la educación económica y ambiental de pobladores, directivos y tomadores de decisiones con vistas a concretar proyectos de desarrollos sostenibles. Se identifica para el estudio hasta la cota cinco, aproximadamente, como el área de mayor interacción entre los subsistemas económico, ambiental y social para la zona costera norte de Camagüey, Cuba. El beneficio económico ambiental supera los 898 millones de pesos, cuyo mayor porcentaje corresponde a los bienes en conservación (45,48 \%). El costo ambiental sobrepasa los 22 millones de pesos, con mayor incidencia del financiamiento para la sostenibilidad seguida por la pérdida del beneficio económico del sistema productivo. La relación beneficio costo exhibe un interesante resultado con 39 pesos por cada unidad del costo ambiental y un valor actual de 3641 millones de pesos.

\section{REFERÊNCIA}

ALDEN, D. Recreational user management of parks: an ecological economics framework. Working Paper no 9707. Centre for Resources and Environmental Studies. Canberra. The Australian National University, 1997.

BARSEV, Rado. Valoración Económica Integral de los Bienes y Servicios Ambientales de la Reserva del Hombre y la Biosfera de Río Plátano. Corrredor Biológico Mesoamericano. Tegucigalpa, 55p. 2002. Disponible en: <http://www.ibcperu.org/doc/isis/8251.pdf >. Acceso en: 12 mayo 2010

BOVARNICK, A.; ALPIZAR, F.; SCHNELL, C. (Eds). La importancia de la biodiversidad y de los ecosistemas para el crecimiento económico y la equidad en América Latina y el Caribe: Una valoración económica de los ecosistemas, Programa de las Naciones Unidas para el Desarrollo, 2010, 357p. 
CERDA, A. Valoración Económica del Medio Ambiente. II Curso Instrumentos de Mercado y Fuentes de Financiamiento para el Desarrollo Sostenible. PNUD, Cartagena de Indias: Colombia, 1-9, julio. 2003. Disponible en: <http://. www.undp.org.cu/eventos/....pdf >. Acceso en: 16 Oct 2008.

DE GROOT, R.; WILSON, M.; BOUMANS, R. A typology for the classification, description and valuation of ecosystem functions, goods and services. Ecological Economics n. 41, p. 393-408. 2002.

DIXON, J.A.; HUFSCHMIDT, M.M. Economic Analysis of Environmental Impacts. London: Earthscan Publications Ltd, 1988.

DOSI, C. Environmental values, valuation methods and natural disaster damage assessment. Environment and Human Settlements Division. Santiago Chile: CEPAL, 2001.

GARCÍA, L.; COLINA, A. Métodos directos e indirectos en la valoración económica de bienes ambientales. Aplicación al valor de uso recreativo del Parque Natural Somiedo. Revista Estudios de Economía Aplicada, v.22, n.3, p. 811-838. 2004.

HERRUZO, A.C. Fundamentos y Métodos para la Valoración de Bienes y Servicios Ambientales. Jornada Temática "Aspectos Medioambientales de la Agricultura". Disponible en: <http://www.libroblancoagricultura.com>, 2002. Acceso en: 14 Sept 2009.

LINDHJEM, Henrik; HU, Tao; MA, Zhong; SKJELVIK, John Magne; SONG, Guojun; VENNEMO, Haakon; WU, Jian; ZHANG, Shiqiu. Environmental Economic Impact Assessment in China: Problems and Prospects. Environmental Impact Assessment Review, United States: Elsevier Inc, v.1, n.27, p.1 - 25, january. 2007.

MOONS, E. Cost-benefit analysis of the location of new forest land. Working Paper $\mathrm{n}^{\circ}$ 2002-05. Katholieke Universiteit Leuven. Belgium, p. 20. 2002.

PEARCE, D., TURNER, K. Economía de los recursos naturales y del medio ambiente. Madrid: Celeste Ediciones, 1995.

PROTOCOLO DE KYOTO. Convenio Marco sobre Cambio Climático de la ONU en la United Nations Framework Convention on Climate Change (UNFCCC), 1997. Disponible en <http://www.unfccc.int/portal_espanol/essential.../kyoto.../3329>. Acceso en: 26 Sept 2009.

SARMIENTO, M.A. Desarrollo de un nuevo método de valoración medioambiental. 2003. 224 p. Tesis (Doctorado en Ciencias) - Departamento de Ingeniería Forestal, Universidad Politécnica de Madrid, Madrid, 2001.

TOMASINI, D. Valoración económica del ambiente. Departamento de Economía, Desarrollo y Planeamiento Agrícola. Facultad de Agronomía. Universidad de Buenos Aires, 2012. Disponible en: <http://www.untrm.edu.pe/.../2012/12/Importancia-de-laVE-Ambiental.pdf>. Acceso en: 16 de Feb 2013. 
VILLA, F.; WILSON, M.; DE GROOT, R.; FARBER, S.; CONSTANZA, R.; BOUMANS, R. Designing an integrated knowledge base to support ecosystem services valuation. Ecological Economics, n. 41, p. 445-456. 2002.

VREEKER, R.; NIJKAMP, P.; TER WELLE, C. A multicriteria decision support methodology for valuating airport expansion plans. Tinbergen Institute Discussion Paper TI 2001-005/3, p. 26. 2001.

WINKLER, R. Valuation of Ecosystem goods and services. Part 1: An Integrated Dynamic Approach. Ecological Economics, v.59, n.22, p. 82-93. 2006. 